\title{
Preliminary Study: Role of the Combination of Spirulina and Morinda Citrifolia in the control of Hyperglycemia in Omani Diabetic Patients
}

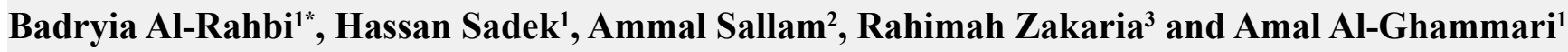 \\ ${ }^{1}$ Department of Health Sciences, Ministry of Health, Oman, Muscat \\ ${ }^{2}$ Research Institute of Ophthalmology, Giza, Egypt \\ ${ }^{3}$ Department of Physiology, School of Medical Sciences, Universiti Sains Malaysia Kubang Kerian, Malaysia
}

*Corresponding author: Badriya Al-Rahbi, Department of Ministry of Health, Institute of Health Sciences, Oman, Muscat, Tel: +0096824560085; Fax: 0096824560384; Ext: 1005; Email: drbadriya.bsc.ihs@gmail.com

\begin{abstract}
Diabetes mellitus is a metabolic disorder characterized by hyperglycemia, hyperlipidemia, hyper aminoacidemia, and hypoinsulinemia that leads to a reduction in both insulin secretion and insulin action.

The combination of Spirulina and Morinda citrifolia as functional supplements are emerging as important therapeutic food. This preliminary study was aimed to evaluate the hypoglycaemic and hypolipidemic effects of combining Spirulina and Morinda citrifolia to subjects with type 1 diabetes mellitus using a daily dose of $4 \mathrm{~g}$ / weight/day tablets for 4 months of Spirulina and 40ml/day for Morinda citrifolia juice with standard insulin treatment in comparison with diabetic subjects receiving type 1 standard diabetes treatment, insulin injections only.

Pre-intervention and post intervention fasting blood glucose levels, glycosylated haemoglobin (HbA1c) levels, and low-density lipoprotein (LDL) serum levels of the diabetic subjects were measured. Four months supplementation of combined Spirulina and Morinda citrifolia resulted in an appreciable lowering of fasting blood glucose blood serum levels. A significant reduction in the HbA1c level was also observed, indicating improved long-term glucose regulation.

These findings suggest the beneficial effect of combining Spirulina with Morinda citrifolia supplements in controlling blood glucose levels and in improving components of the lipid profile of Omani subjects with type 1 diabetes mellitus.
\end{abstract}

Received Date: October 26, 2016

Accepted Date: December 12, 2016

Published Date: December 19, 2016

Citation: Al-Rahbi, B., et al. Preliminary Study: Role of the Combination of Spirulina and Morinda Citrifolia in the control of Hyperglycemia in Omani Diabetic Patients. (2016) Int J Food Nutr Sci 3(2): 385-388.

DOI: $10.15436 / 2377-0619.16 .1185$

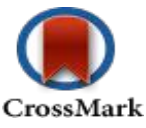

Keywords: Spirulina; Morinda citrifolia; Diabetes mellitus and blood glucose

\section{Introduction}

Diabetes mellitus is a group of metabolic diseases characterized by hyperglycemia resulting from defects in insulin secretion, insulin action, or both. There are two types of diabetes, which are namely the type 1 (insulin-dependent diabetes) where the body does not produce insulin and type 2 (insulin-independent diabetes) where the body does not produce enough insulin. Approximately $90 \%$ of all cases of diabetes worldwide are type $2^{[1]}$.

Diabetes is becoming a common disease in the Middle
East region as the incidence of type 1 diabetes ranges from 2.62$20.18 / 100,000 /$ year and the percentage of type 1 diabetes is 4.6 to $40 \%$ in the region ${ }^{[1]}$.

Plants have always been a good source of drugs, and many of the currently available drugs are derived directly or indirectly from them. Herbs play a big role in managing several diseases including diabetes. Examples of herbs used as anti-diabetic agents are the following, Ganoderma lucidium, Morinda citrifolia, Spirulina, Allium sativum, Eugenia jambolana, mo- 
mordica charantia ocimum sanctum, phyllanthus amarus, tinospora cordifolia and trigonella foenum graecum ${ }^{[2]}$.

The Ganoderma lucidum, which is an example of a herbal anti-diabetic agent, is a large dark mushroom, which grows in six colours; blue, red, yellow, purple, black and white, each has different names and characteristics ${ }^{[3]}$. An animal experiment was done by Xiao and colleague ${ }^{[4]}$ to investigate the hypoglycemic effects and action of Ganoderma lucidum polysaccharides (GLP) on diabetic mice. Results showed that GLPs received mice had significantly decreased the fasting serum glucose compared with the diabetic control group ${ }^{[4]}$.

Morinda citrifolia, which belongs to the family Rubiaceae; are small evergreen trees that grow in tropical climate zone $^{[5]}$. The leaves are about 8 inches long and are oval, the tubular flowers are white and the fruit is 3 to 4 inches in diameter with a warty, pitted surface ${ }^{[6]}$.

Morinda citrifolia can be found in India, Southeast Asia, Australia, Polynesia and Hawaii. The fruit is commonly known as "Noni" or "Indian Mulberry"; in Australia it is called "cheese fruit" and in Japan "Yaeyama-aoki"[7].

In the Pacific Islands "noni"- Morinda citrifolia is used by the Polynesians in folk medicine. All parts of the plants have been used, including leaf, fruit, flower, roots, bark and seed. The juice extracted from Morinda citrifolia fruit possesses an anti-oxidative activity. Kamiya et al. ${ }^{[8]}$ showed that the methanol extracts and ethyl acetate-soluble phases from Morinda citrifolia fruit inhibited copper-induced LDL (low-density lipoprotein) oxidation. Previously Wang et al. ${ }^{[9]}$ showed that extracts from Morinda citrifolia induced dose-dependent inhibition of the oxidant substances produced by lipid hydrogen peroxide or superoxide anion radicals.

A detailed study of the nutritional composition of $\mathrm{Mo}$ rinda citrifolia juice has been analyzed. Results showed that it contains glucose and fructose ( $3-4 \%$ each), protein $(0.2-0.5$ $\%)$, lipids $(0.1-0.2 \%)$ and vitamin $\mathrm{C}$. The potassium content is relatively high (30 - $150 \mathrm{ppm})$, followed by calcium, sodium and magnesium $^{[5]}$.

On the other hand, Spirulina is a blue-green microalgae that grows in tropical salt lakes. It is a multicellular, filamentous cyanobacteria belonging to the algae class cyanophyta ${ }^{[10]}$. It is produced primarily from two species of cyanobacteria: Arthrospiraplatensis and arthrospira maxima classified into the genus Arthrospira ${ }^{[11]}$. Spirulina is proven to be a rich source of protein and vitamins, especially vitamin $B_{12}$, minerals, carotenoids, and phycocyanins $^{[12]}$.

Beyond nutritional value, certain species of Spirulina have shown therapeutic properties such as metabolic, anti-viral, liver-protecting and blood-vessel relaxing effects; anti-cancer, anti-inflammatory and antioxidant properties ${ }^{[11]}$. A human study was done by Mani etal. ${ }^{[1]}$ to observe the long-term effect of spirulina in diabetic patients. After 2 months of treating patients with Spirulina only, the results showed significant reduction of blood sugar levels and glycosylated serum protein levels, confirming the hypoglycaemic effect of spirulina. Kim and Park ${ }^{[13]}$ asserted that Spirulina can be used as dietary supplementation in nutritionally vulnerable groups, Korean elder lies, to improve nutritional and health status and prevent chronic diseases such as hyperlipidaemia ${ }^{[13]}$.

Kusirisin et al. ${ }^{[14]}$ compared between morinda citrifolia and other natural plants such as Kaempferia parviflora and re- ported that morinda citrifolia had the lowest total poly phenol and tannin content.

Considering previous studies, we hypothesized that the combination of Spirulina and Morinda citrifolia might give stronger anti-diabetic effects. Our study aimed to report the antidiabetic and lipid profile effects of the combination of Spirulina and Morinda citrifolia from four diabetic Omani patients.

\section{Materials and Methodology}

Four Omani diabetic patients that were between 22 - 35 years of age, who had been diabetic for 12 years of duration, had daily administered the combined herbal intervention for 4 months $4 \mathrm{~g}$ /day of Spirulina tablets and $40 \mathrm{ml} /$ day of Morinda citrifolia juice with standard insulin treatment. Pre-intervention and post intervention fasting blood glucose levels, glycosylated haemoglobin (HbA1c) levels, low-density lipoprotein (LDL) and body weight were reported in all the subjects. Other four Omani diabetic patients with same age range and duration of the disease receiving type 1 standard diabetic treatment, insulin injections only.

\section{Statistical Analysis:}

The statistical SPSS program (version 20.0) was used for all the analysis. The data were analyzed by one-way ANOVA and two-way ANOVA. All the results are expressed as mean \pm standard error of the mean (SEM), and with the level of significance set at $\mathrm{P}<0.05$.

\section{Results}

Table (1) shows characteristics of the subjects. Two females and two males with duration of 12 years of diabetes for group 1 as a control group. There were three females and one male with duration of diabetes for 12 years for group 2 receiving the intervention.

Table1: Characteristics of the investigated subject.

\begin{tabular}{|l|c|c|}
\hline & Group 1* & Group 2\# \\
\hline Female & 2 & 3 \\
\hline Male & 2 & 1 \\
\hline
\end{tabular}

Duration of diabetes 12years 12years

Group $1^{*}=$ diabetic patients receiving type 1 standard diabetic treatment- insulin injections; as a control group

Group $2 \#=$ diabetic patients receiving herbal anti-diabetic intervention

Table 2: Body weights of subjects investigated.

\begin{tabular}{|l|l|}
\hline Groups (Kg) & Body weight \\
\hline Group 1 & $49.75 \pm 2.00$ \\
\hline Group 2: & Before pre-intervention $49.96 \pm 1.80$ \\
\hline Group 2: & 4 months post intervention $50.50 \pm 2.10$ \\
\hline
\end{tabular}

No significant change was found in the body weights of the subjects of group 2 post intervention compared to the pre-intervention weights $\mathrm{P}=0.07$. Also, there was no significant change as well when compared to the control group - group 1, P $=0.06$. 
Post intervention, fasting blood glucose serum levels of group 2 subjects, that used the combination of spirulina and morinda citrifolia, were significantly lower $(\mathrm{P}<0.002)$ as compared to pre-intervention measurement of those patients and also in comparison to fasting blood glucose serum levels post-treatment for the control group- group $1(\mathrm{P}<0.001)$ as shown in figure 1 .

Blood glucose serum levels

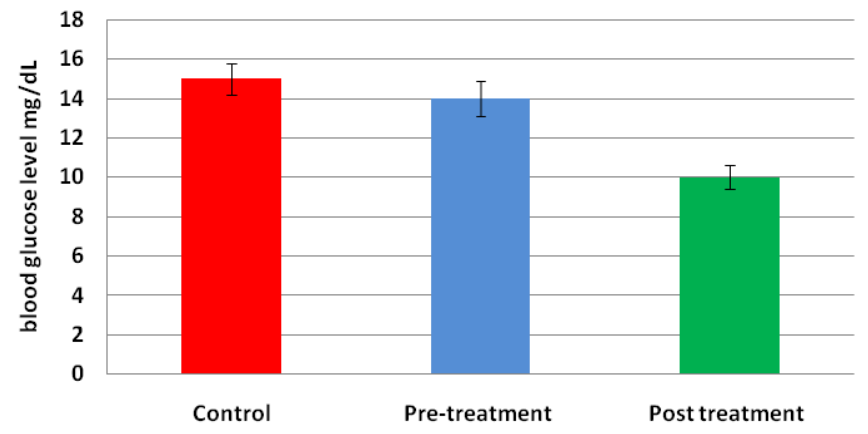

Figure 1: Fasting blood glucose serum levels.

Post intervention glycosalated haemoglobin serum levels of the group 2 subjects treated with a combination of spiruli$n a$ and morinda citrifolia were significantly lower $(\mathrm{P}<0.05)$ as compared to pre-intervention measurement of those patients and when compared to the control group, which is group 1 .

Table 3: Glycsolated haemoglobin serum levels.

\begin{tabular}{|l|l|}
\hline Groups \% & HbA1C \\
\hline Group 1 & 6.4 \\
\hline Group 2: & Pre intervention 6.5 \\
\hline Group 2: & Post intervention 5.8 \\
\hline
\end{tabular}

As shown in Table 4, low-density lipoprotein (LDL) of group 2 subjects that received the combination of Spirulina and Morinda citrifolia were significantly lower $(\mathrm{P}<0.019)$ as compared to pre-intervention measurement of group 2 patients and also significantly lower when compared to control group, group $1(\mathrm{P}<0.018)$.

Table 4: Low-density lipoprotein (LDL) serum levels.

\begin{tabular}{|l|l|}
\hline Groups $(\mathrm{mg} / \mathrm{dL})$ & LDL \\
\hline Group 1 & $153.00 \pm 4.12$ \\
\hline Group 2: & Pre intervention $156.00 \pm 3.82$ \\
\hline Group 2: & Post intervention $145.00 \pm 2.40$ \\
\hline
\end{tabular}

\section{Discussion}

Diabetes mellitus (DM) complications not only reduce the quality and length of life but also are responsible for enormous healthcare costs. More than $50 \%$ of the 194 million people suffering from diabetes mellitus globally do not know that they have the disease. The majority of the undiagnosed and untreated people live in the developing world.

Diabetes mellitus (DM) is suspected based on symp- toms such as polyuria, polydipsia, polyphagia, weight loss, recurrent signs of infections. The DM is also associated with micro vascular complications involving retinopathy, nephropathy, and neuropathy. As a usual practice with the health care facilities of the Ministry of Health, Oman, DM is diagnosed at and registered with the Primary Health Centres (PHC), where the patient is followed up in order to monitor glucose control through a team involving a physician to manage medically, to prescribe medication and referring to other medical speciality should a patient requires further attention from secondary or tertiary hospital facilities $^{[15]}$

A recent study showed that an estimated 10-year risk for cardiovascular disease among Omani adults with diabetes increased significantly between 1991 and 2008 in Oman. Focused public health initiatives, involving recognised interventions to address behavioural and biological risks, should be a real national priority ${ }^{[16]}$.

The present invention provides individuals with DM the opportunity to safely and conveniently take measures to reduce the likelihood of contracting diabetes-associated complications, as well as the opportunity to safely treat existing diabetes conditions using a natural formulation and method of treatment rather than more invasive treatment with insulin injections.

The previous study concluded that spirulina is a safe food supplement without significant side-effects. Kusirisin et al. ${ }^{[14]}$ stated that Morinda citrifolia had the lowest total polyphenol and tannin content, which is known to have an antioxidant activity, includes chelating free radicals preventing lipid per oxidation.

We hypothesized that combining Morinda citrifolia and Spirulina will give better anti-diabetic effects. Our preliminary study aimed to analyze the effects of the combinations of the two natural products of Morinda citrifolia and Spirulina.

Although Panam et al. ${ }^{[17]}$ showed in their study a significant improvement in the body weight following Spirulina supplementation. In the present study, we found that the body weights of these Omani diabetic patients were not significantly changed when compared to the pre-intervention weights. A reasonable explanation could be that period of intervention was not enough to improve the weight in these subjects.

However, our analysis showed that after we tried on the combination of Morinda citrifolia and Spirulina to exert insulin-producing organ to secrete native insulin for glucose control, serum levels of glucose and glycosylated haemoglobin were significantly lower as compared to the pre-intervention measurement of those patients, indicating improved long-term glucose regulation.

The results of reduced blood glucose could be due to the ability of those supplementary in delaying the absorptions of glucose via inhibitions of $\alpha$-glucosidase, which is located in the brush border of the small intestine and normally helps to breakdown starch and disaccharides to glucose ${ }^{[18]}$.

This study also reported that the LDL of these diabetic patients was significantly decreased when compared to the pre-intervention levels of LDL. As glucose attaches to LDLs in the blood so, LDLs coated with glucose will stay in the bloodstream for a longer duration.

The mechanism of improvement for the lipid profile was reported earlier through animal experiments by Han LK et al. ${ }^{[5]}$ which had stated that glycolipid H-b2 isolated from Spiruli- 
$n a$ was able to inhibit pancreatic lipase activity in a dose-dependent manner and able to reduce postprandial TG levels. Besides, it was found that phycocyanin also inhibits pancreatic lipase.

Although these measures help in controlling DM, but if the underlying condition progress and existing measures do not control glucose level, DM condition remains obscure on a long run thus giving rise to adverse morbidity. Clinical trials involving a larger sample size will be needed to carry out additional efforts to confirm these results.

\section{Conclusion}

In conclusion, our study sheds the light on the effects of the combination of Morinda Citrifolia and Spirulina as strong anti-diabetics. The study brought in favourable effect on blood glucose and blood lipids-LDL in Diabetic Omani patients. Our results also suggest that Spirulina and Morinda citrifolia are capable agents and should be considered as useful food supplements for the management of diabetes. Further studies with a larger sample size and for a longer duration are required to ascertain the mechanism of combined Spirulina and Morinda citrifolia actions in more detail and its antioxidant capacity and immune variables.

\section{References}

1. Mani, U.V., Desai, S., Iyer, U. Studies on the long-term effect of Spirulina supplementation on serum lipid profile and glycosalted proteins in NIDDM patients. (2000) J Nutrac Funct Med Foods 2(3): 25-32.

2. Adeghate, E., Schattner, P., Dunn, E. An Update on the Etiology and Epidemiology of Diabetes Mellitus. (2006) Ann N YAcad Sci 1084: 1-29.

3. Sissi, W.G., Yuen, J., Buswell, J., et al. Ganoderma lucidum (Lingzhi or Reishi). (2011) Herbal Medicine: Biomolecular and Clinical Aspects. $2^{\text {nd }} \mathrm{Ed}$.

4. Xiao, C., Wu, Q.P., Cai, W., et al. Hypoglycemic effects of Gandoerma lucidum polysaccharides in type 2 diabetic mice. (2012) Arch Pharma Res 35(10): 1793-1801.
5. Han, L.K., Li, D.X., Xiang, L., et al. Isolation of pancreatic lipase activity-inhibitory component of Spirulina platensis and it reduce postprandial triacylglycerolemia. (2006) Yakugaku Zasshi 126(1): 43-49.

6. Potterat, O., Hamburger, M. Morinda citrifolia (Noni) Fruit - Phytochemistry, Pharmacology, Safety. (2007) Planta Med 73(3): 191-199.

7. Nayak, S.B., Marshall J.R., Isitor G., et al. Hypoglycemic and Hepatoprotective Activity of Fermented Fruit Juice of Morinda citrifolia (Noni) in Diabetic Rats. (2011) J Evid-Based Compl Alter Med (2011): 5 .

8. Kamiya, K., Tanaka, Y., Endang, H., et al. Chemical Constituents of Morinda citrifolia Fruits Inhibit Copper-Induced Low-Density Lipoprotein Oxidation. (2004) J Agric Food Chem 52(19): 5843-5848.

9. Wang, M., Kikuzaki, H., Csiszar, K., et al. Novel Trisaccharide Fatty Acid Ester Identified from the Fruits of Morinda Citrifolia (Noni). (1999) Agricul Food Chem 47(12): 4880-4882.

10. Mazokopakis, E.E., Starakis, I.K., Papadomanolaki, M.G., et al. The hypolipidaemic effects of Spirulina (Arthrospira platensis) supplementation in a Cretan population: a prospective study. (2013) J Sci Food Agric 94(3): 432-437.

11. Kamiya, K., Hamabe, W., Harada, S. et al. Chemical constituents of Morinda citrifolia roots exhibit hypoglycemic effects in streptozotocin-induced diabetic mice. (2008) Biol Pharm Bull 31(5): 935-938.

12. Layam, A., Reddy, C.L.K. Antidiabetic property of Spirulina. (2006) Diab Croat 35(2): 29-33.

13. Kim, Y., Park, Y. The effect of Spirulina on lipid metabolism, antioxidant capacity and immune function in Korean elderly. (2003) Korean J Nutr 36(3): 287-297.

14. Kusirisin, W., Srichairatanakool, S., Lerttrakarnnon, P., et al. Antioxidative activity, polyphenolic content and anti-glycation effect of some Thai medicinal plants traditionally used in diabetic patients. (2009) Med Chem 5(2): 139-147.

15. Alyaarubi, S. Diabetes care in Oman: obstacles and solutions. (2011) Sultan Qaboos Univ Med 11(3): 343-348.

16. Al-Lawati, J., Morsi, M., Al-Riyami, A., et al. Trends in the risk for cardiovascular disease among adults with diabetes in Oman. (2015) Sultan Qaboos Univ Med J 15(1): 39-45.

17. Panam, P., Uliyar, M., Uma, I. Role of Spirulina in the control of glycemia and lipidemia in type 2 diabetes mellitus. (2004) J Med Food 4(4): 193-199.

18. Senthil, N., Balu, P. M., Murugesan, K. AntiHyperglycemic effect of Spirulina, Insulin and Morinda citrifolia against Streptozotocin induced diabetic rats. (2013) Int J Curr Microbiol App Sci 2(10): 537-559.
Online ISSN: $2377-0619$

Journal Title: International Journal Food and Nutritional Science Journal Short Name: Int J Food Nutr Sci
Ommega Online Publishers

E-mail: foodscience@ommegaonline.org

Website: www.ommegaonline.org 